\title{
Binaphthalene-based polymer membranes with enhanced performance for solvent-resistant nanofiltration
}

\author{
Marloes Thijs ${ }^{a}$, Cédric Van Goethem ${ }^{b}$, Ivo F.J. Vankelecom ${ }^{b}$ and Guy Koeckelberghs ${ }^{a *}$ \\ aLaboratory for Polymer Synthesis, Department of Chemistry, KU Leuven, Celestijnenlaan 200F, B-3001 \\ Heverlee, Belgium \\ ${ }^{b}$ Membrane Technology Group - cMACS; Department of Microbial and Molecular Systems, KU Leuven, \\ Celestijnenlaan 200F, B-3001 Heverlee, Belgium
}

Solvent-resistant nanofiltration, interfacial polymerization, adjustable molecular structure, polymers of intrinsic microporosity

\section{ABSTRACT}

To improve the performance of the currently available solvent-resistant nanofiltration (SRNF) membranes, a new, unique approach was developed. In this approach, the concept of PIMs is combined with the fabrication of thin film composite membranes by interfacial polymerization (IFP) ànd the possibility to tune the molecular structure of the monomers at wish. This allows to obtain a thin active layer with a high free volume and good permeance for solvents while realizing a high solute retention. The influence of the dihedral angle of the binaphthalene-based di(acid chloride) and the influence of the number and position of the amine functionalities of the amine monomer on the membrane performance is studied. By combining a binaphthalene-based di(acid chloride) with various amine monomers containing 2.5-4 amine functions via IFP on a crosslinked PI (Matrimid) support, the permeance of acetonitrile (ACN) could be increased by a factor of 20 in comparison to standard TFC-membranes consisting of TMC and MPD, at a $99 \%$ retention of Rose Bengal (RB, $1017 \mathrm{~g} / \mathrm{mol}$ ). The highest permeance in combination with the best retention were obtained when using the methyl-linked binaphthalene di(acid chloride), which has the smallest dihedral angle.

\section{Introduction}

Over the past two decades, solvent resistant nanofiltration (SRNF) or organic solvent nanofiltration (OSN), has gained much attention as it opens up the possibility to reduce energy consumption and solvent waste.[1,2,3] This process typically consumes an order of magnitude less energy than conventional processes, since membrane processes only require pump energy to apply the required pressure difference.[3,4,5] SRNF can be used as an alternative to or in combination with traditional purification techniques such as distillation, evaporation, adsorption, extraction and preparative chromatography and can be applied in the chemical, food, pharmaceutical, oil and gas industry.[1,3,6,7,8,9,10,11,12] Apart from an intrinsic chemical stability, an important requirement of the membranes is a high permeance combined with a high retention.[13] However, a trade-off still exists between permeance and retention,

\footnotetext{
SRNF, solvent resistant nanofiltration; GC-MS, gas chromatography mass spectrometry; MPD, m-phenylene diamine; TMC, trimesoyl chloride; SEM, scanning electron microscopy; TEM, transmission electron microscopy; PIM, polymer of intrinsic microporosity; BN, binaphthalene; RB, Rose Bengal; TEA, trimethylamine; ACN, acetonitrile; SDS, sodium dodecyl sulfate; TFC, thin film composite; $C D$, circular dichroism; TA, triamine/tetraamine; AC, acid chloride; CA, carboxylic acid; PI, polyimide; PA, polyamide; $\mathrm{MO}$, methyl orange; XL-PI, crosslinked polyimide; IFP, interfacial polymerization.

*Corresponding author. Email address: guy.koeckelberghs@kuleuven.be
} 
which restricts the application of SRNF.[6,14,15] To overcome this, many attempts have been made to improve permeance while maintaining a high solute retention.[16] An increase in permeance can be obtained in two ways: (1) by reducing the thickness of the active layer and (2) by increasing the free volume of the selective layer resulting in an accelerated transport through the membrane. $[7,17,18]$ Nanofiltration membranes were first developed for aqueous applications and are commonly prepared via solution processing of polymers and interfacial polymerization (IFP).[2,6,7,19,20,21] Additional processing steps such as crosslinking are often necessary to make the membranes applicable for SRNF.[8,22] Stateof-the-art membranes for nanofiltration are thin film composite membranes (TFC) comprising of a thin active top-layer formed on top of a porous support (Figure 1).[23,24,25] The support typically consists of 2 layers: a 'non-woven', typically made from polyethylene or polypropylene, and a porous support, usually prepared via phase inversion.[2] The second layer allows the formation of a thin, defect-free top-layer. The advantage of TFC membranes is that the formation of the thin top-layer is independent from the support layer.[3,23,24] In general, the top-layer is formed via IFP, in which a polymer network is formed at the interface of two immiscible solvents in which the monomers are dissolved.[25] One monomer, normally a diamine monomer, diffuses from the water to the organic phase, containing the tri(acid chloride), where the PA top-layer is formed. [13]

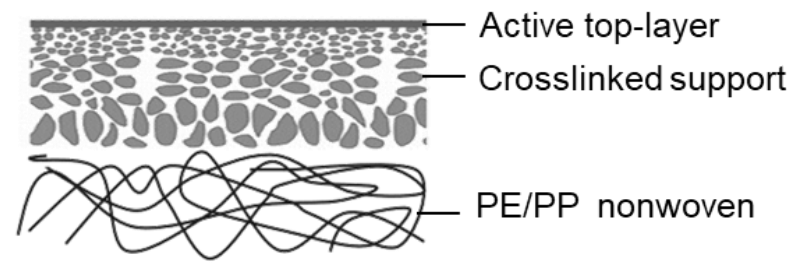

Figure 1. Schematic representation of a TFC membrane consisting of a top-layer and a bi-layered substructure.

Typically, a thin, i.e. $<100 \mathrm{~nm}$, PA film is formed when using m-phenylene diamine (MPD) and trimesoyl chloride (TMC). $[13,18,27]$ However, this gives rise to a thin, dense and fairly polar layer with rather low solvent permeances.[6,20] In 2004, a new type of polymer was developed by McKeown and Budd, i.e. polymers of intrinsic microporosity (PIMs) (Figure 2). $[28,29,30]$ These polymers have a contorted and rigid backbone leading to inefficient stacking in the solid state, creating interconnecting voids of less than $2 \mathrm{~nm}$ that behave as micropores.[31] PIMs are soluble in solvents and can therefore readily be processed by solvent-based techniques.[32] They can easily be formed into robust films, coatings or fibers, and are promising materials for gas applications.[31] PIMs can be applied in SRNF after modifying the polymers via post-polymerization modifications, such as crosslinking.[33] However, this implies the need for an extra step.[22] In 2016, an IFP was developed with enhanced microporosity based on the concept of PIMs.[34] Highly crosslinked polyarylate thin films of enhanced microporosity were obtained with outstanding performance in SRNF. The microporosity originates form the presence of a spirocenter in the 5, 5',6,6'-tetrahydroxy-3,3,3',3'-tetramethylspirobisindane monomer, as commonly used for the synthesis of PIMs for gas separation.[35] 




Figure 2. Structure of PIM-1. The contorted structure is induced by the presence of a spiro-center. [35]

The disadvantage is that the structure of this monomer cannot be adjusted. Similar to this, a poly(esteramide) was made using two bisphenols with rigid, contorted backbones in combination with piperazine as aqueous-phase monomers, employed for IFP with trimesoyl chloride in cyclohexane to synthesize TFC membranes.[16] The water flux was increased two- to three-fold while maintaining almost the same retention (>99.2\% for $\mathrm{Na}_{2} \mathrm{SO}_{4}$ ). However, once again the disadvantage is that the structure of the bisphenols cannot be adjusted. Furthermore, Duong et al. made thin porphyrin composite membranes with an enhanced organic solvent transport. The hybrid polyamide films were formed by IP of 5,10,15,20(tetra-4-aminophenyl)porphyrin/m-phenylene diamine (MPD) mixtures with trimesoyl chloride. By incorporating porphyrin, which is a non-planar molecule, a polyamide film with an increased free volume was obtained. Increasing the porphyrin/MPD ratio led to an increase in permeance but a decrease in retention. [36] Y-J. Tang et al. succeeded in tailoring the backbone stiffness by combining piperazine (PIP) and bisphenol F (BPF) in the aqueous phase with trimesoyl in the organic phase. In this way, the backbone stiffness and the microporosity degree could be enhanced resulting in an increased pure water flux without compromising salt retention.[37] Based on this study, Tang et al. developed a PIP/BPF/2,2'-bis(1hydroxyl-1-trifluoromethyl-2,2,2-triflutoethyl)-4,4'-methylene (BHTTM) hollow-fiber membrane possessing both a high water flux and excellent chlorine resistance, maintaining an enhanced backbone stiffness and microporosity. [38] Since IFP allows synthesis of thin selective layers and since the use of PIMs has been shown to be very promising for SRNF as well, the aim of this research is to combine these two concepts while implementing monomers that enable fine-tuning of the molecular structure. The tunable monomer is a bridged binaphthalene di(acid chloride) (Figure 3) of which the alkyl linker can be adjusted. This monomer is combined with several amine monomers in which the number of amine functional groups is varied. In this way, it becomes possible to study the influence of the molecular structure on the macromolecular structure of the polymer membrane and thus the membrane performance.

\section{Experimental}

\subsection{Materials and methods}

Materials. All solvents were purchased from Fisher, VWR or Chem-Labs. All reagents were purchased from Acros Organics, Sigma-Aldrich, Alfa Aeser, Thermo Fisher, AK Scientific or J\&K Scientific. PI (Matrimid 5218) was purchased from Huntsman (Switzerland) and used after drying at $100{ }^{\circ} \mathrm{C}$. All reactions using moisture- or oxygen-sensitive reagents were done in oven-dried glassware under nitrogen atmosphere unless specified otherwise. Distilled water was used for the support synthesis and the water filtrations.

Preparation of the crosslinked PI support. A $14 \mathrm{wt} \%$ solution of Matrimid was dissolved in a 3:1 w:w ratio of NMP/THF. The solution was stirred overnight and left standing to degas for $24 \mathrm{~h}$. Afterwards, the 
solution was cast, using an automatic film applicator (Braive Instruments, Belgium), onto a PE/PP nonwoven fabric (Novatexx 2471), taped on a glass plate. This was done in a temperature and humidity controlled room $\left(16^{\circ} \mathrm{C}, 30 \pm 10 \%\right.$ humidity). The casting knife had an opening of $250 \mu \mathrm{m}$ and a speed of $3.8 \mathrm{~cm} / \mathrm{s}$.[39] After an evaporation step of $30 \mathrm{~s}$, the cast film was immersed in a coagulation bath containing distilled water with a temperature of around $18{ }^{\circ} \mathrm{C}$. The PI supports were stored in distilled water for $24 \mathrm{~h}$, after which they were soaked in methanol for about 3-4 hours (after around 2 hours the methanol was renewed, per $0.1 \mathrm{~m}^{2}$ of membrane around 3 to $4 \mathrm{~L}$ of $\mathrm{MeOH}$ was used) to exchange the water before crosslinking in a methanol solution containing $5 \mathrm{wt} \%$ hexanediamine (HDA) for $1 \mathrm{~h}$ at room temperature.[40] Finally, the crosslinked PI supports were rinsed with methanol (the solvent was renewed 2-3 times, in total the support was kept in methanol for 3-4 hours) and stored in distilled water until further use. As a result of the excessive rinsing and the contact time of the support with water, the probability of unreacted HDA being present in the support is assumed to be negligible. This is important since HDA would otherwise react with trimesoyl chloride instead of the various amine monomers used. A SEM cross-section image of the support, with a thickness of around $80 \mu \mathrm{m}$, can be seen in Fig. S1. The support has a thickness of around $80 \mu \mathrm{m}$ and a test filtration was standardly performed on the support using RB in water (see supporting info).

Preparation of TFC Membranes. To avoid degradation of the free amine monomers, the amine solution was freshly prepared for each membrane batch. To further avoid degradation, the distilled water used for the solution was sonicated and purged with argon to remove the oxygen present in the water. Moreover, argon was used, since it creates a blanket of inert gas over the solution again protecting the amine from oxygen in the air. To convert the amine salt to the free amine monomer, triethylamine (TEA) was added to the water phase. Immediately after the addition of TEA, the PI support was immersed in the amine solution for $5 \mathrm{~min}$ in a purpose-built reservoir, designed in such a way that contact between the solution and the air is limited. Afterwards, the support was removed from the solution, with a wiper residual amine solution was removed and the top of the support was contacted with the organic phase. This phase consists of the bridged binaphthalene di(acid chloride) dissolved in toluene. The two phases are kept in contact for $1 \mathrm{~min}$, after which the organic solution was removed, the membrane was rinsed with clean toluene, left to dry in the air for $1 \mathrm{~min}$ and placed in a beaker with distilled water.

Membrane Characterization. The surfaces of the prepared membranes were visualized using a scanning electron microscope (SEM, JEOL JSM-6010LV). Before analysis, all samples were coated with a thin Au/Pd layer (1.5-2 nm) by a JEOL JFC-1700 Auto Fine Coater to minimize sample charging. The SEM was operated at an acceleration voltage of $10 \mathrm{kV}$. The thickness of each membrane was determined using Transmission Electron Microscopy (TEM). To obtain suitable sections for TEM analysis the membranes were embedded in an epoxy resin (Araldite, Polyscience). Sections of the embedded membranes with a thickness of $70 \mathrm{~nm}$ were cut using a microtome (Reichert Ultra-cut E) and transferred to a $\mathrm{CU}$ TEM grid. TEM images were acquired using a JEOL ARM-200F probe aberration corrected TEM operated at $200 \mathrm{kV}$.

Membrane Performance. The performance of the membranes was determined using a dead-end highthroughput filtration setup containing 16 positions with an active filtration area of $0.000177 \mathrm{~m}^{2}$ each.[41] A feed solution of $35 \mu \mathrm{M}$ Rose Bengal (RB, $1017 \mathrm{Da}$ ) or Methyl Orange (MO, $327 \mathrm{Da}$ ) in ethanol, ACN or water was used and stirred at $400 \mathrm{rpm}$ to minimize concentration polarization. From every membrane, three coupons were cut and tested to check the reproducibility and to determine the standard deviation. Feed pressure was kept constant at 15 bar. The first obtained permeate was discarded to allow the membrane to reach steady state. At least 2 vials were collected from every membrane coupon to confirm 
that steady state was reached. The collected volume was $5-20 \mathrm{~mL}$ per vial depending on the permeance of each coupon. The permeance $\left(P, L / \mathrm{m}^{2}\right.$.h.bar) was calculated using the following equation

$$
\mathrm{P}=\frac{\mathrm{V}}{\mathrm{A} \cdot \Delta \mathrm{P} \cdot \Delta \mathrm{t}}
$$

with $\mathrm{V}$ the permeate volume, $\Delta \mathrm{t}$ the permeate collection time, $\mathrm{A}$ the effective membrane area and, $\Delta \mathrm{P}$ the applied pressure. The retention $(R)$ was determined using

$$
\mathrm{R}=\left(1-\frac{\mathrm{C}_{\mathrm{p}}}{\mathrm{C}_{\mathrm{f}}}\right) \cdot 100 \%
$$

with $C_{p}$ the permeate solute concentration and $C_{f}$ the feed solute concentration. The solute concentration in the feed and permeate were determined using a Shimadzu UV-1800 UV-Vis spectrophotometer.

Reactivity study. The reactivity of the various amine monomers was determined using gas chromatography-mass spectroscopy (GC-MS, Thermo Scientific ITQ 900 GC/MS combined with a Thermo Trace GC, equipped with a Restek Rxi ${ }^{\circledR}-5 \mathrm{~ms}$ ( $30 \mathrm{~m}, 0.25 \mathrm{mmID}, 0.25 \mu \mathrm{m}$ df) column). For this, each amine was reacted with benzoyl chloride in presence of 1,3,5-trimethoxybenzene as internal standard and chloroform was used as solvent. Initially, an optimum was determined in the ratio of benzoyl chloride to 1,3,5-trimethoxybenzene that could be measured via GC-MS. Based on this optimal benzoyl chloride concentration, the amine concentrations were calculated. To perform the actual measurement, the amine monomer and TEA were dissolved in chloroform, after which the solution was sonicated and filtered over a $0.45 \mu \mathrm{m}$ filter. Subsequently, a stock solution was made from benzoyl chloride with 1,3,5trimethoxybenzene in chloroform. $1 \mathrm{~mL}$ of the benzoyl chloride solution was then added to the amine solution. After $4 \mathrm{~min}$, the reaction mixture was injected into the GC-MS. By dividing the measured peak areas, the ratio of benzoyl chloride to 1,3,5-trimethoxybenzene was determined. The conversion (c) of benzoyl chloride was calculated using following equation:

$$
c=\frac{X_{i} / S_{i}}{X_{f} / S_{f}} \cdot 100 \%
$$

with $X_{i}$ and $S_{i}$ the initial peak area of benzoyl chloride and 1,3,5-trimethoxybenzene, respectively. $X_{f}$ and $S_{f}$ are the final peak areas of benzoyl chloride and 1,3,5-trimethoxybenzene, respectively.

\section{Results and discussion}

\subsection{Design of materials}

To ensure that the polymer network has a rigid and contorted structure, it was decided to work with bridged binaphthalene monomers (1L-BN-AC and 3L-BN-AC, Figure 3) in combination with various, rigid amine monomers (Figure 4). Binaphthalene-based polymers have already been used for nonlinear optics, asymmetric catalysis and chiroptical sensing. $[39,40,41,42,43]$ The choice for the methyl- and propyl-linked binaphthalene di(acid chloride) (1L-BN-AC and 3L-BN-AC) is based on earlier research performed by W. Dujardin et al. and M. Mulunda et al.[44,45] By using circular dichroism (CD) and computational simulations, they were able to demonstrate that the methyl- and propyl-bridged binaphthalene monomers showed the largest difference in dihedral angle. The simulation calculated a dihedral angle of $49.1^{\circ}$ for a methyl-bridged binaphthalene and a dihedral angle of $65.6^{\circ}$ for a propyl-bridged binaphthalene.[48] Therefore, these are the most appropriate structures to study the influence of the 
dihedral angle, and hence the influence of the tilting degree in the structure, on the performance of the membranes<smiles>COCOc1c(C(=O)Cl)cc2ccccc2c1-c1cc2ccccc2cc1C(=O)Cl</smiles><smiles>CCOc1c(C(=O)Cl)cc2ccccc2c1-c1c(OCCCO)c(C(=O)Cl)cc2ccccc12</smiles>

Figure 3. Structure of the binaphthalene di(acid chloride) with a methyl linker (left, $1 L-B N-A C$ ) and a propyl linker (right, $3 L-B N-$ $A C)$.

To obtain a rigid polymer structure with a limited freedom of movement, thus ensuring a high porosity, amine monomers with a rigid phenyl core were used. Except for 1,3,5-benzenetriamine.3HCl $(1,3,5$ TA.3HCl), all amines were commercially available as a salt.

\subsection{Monomer synthesis and characterization}

Two new binaphthalene-based monomers, 1L-BN-AC and 3L-BN-AC (Figure 3), were synthesized (Scheme 1; see supporting info for the reaction procedure). The binaphthalene di(acid chloride) was formed starting from the commercially available (S)-1,1'-Binaphthalene-2,2'-diol. First, the alkyl linker was introduced using dibromomethane and dibromopropane for the formation of the methyl- and propylbridged binaphthalene monomers (1L-BN/3L-BN), respectively.[49] Then, ortho-lithiation, followed by trapping with $\mathrm{CO}_{2}$ and acidification led to the formation of the di(carboxylic acid) (1L-BN-CA/3L-BN-CA).



Figure 4. Structures of the used amine monomers. From left to right: 1,2,4-benzenetriamine.dihydrochloride (1,2,4TA.2HCl), mphenylenediamine (MPD), 1,3,5-benzenetriamine.trihydrochloride $\quad(1,3,5 T A .3 \mathrm{HCl})$ and $1,2,4,5$ benzenetetraamine.tetrahydrochloride $(1,2,4,5 T A)$

For the ortho-lithiation, the reaction conditions were based on the synthesis described by Wu et al.[50] When using the described conditions, only a yield of $19 \%$ was obtained. Therefore, the used solvent mixture was changed from $\mathrm{Et}_{2} \mathrm{O}: \mathrm{THF}$ to $\mathrm{Et}_{2} \mathrm{O}: \mathrm{DME}$. As in the original procedure, DME was added after 3 hours. The reaction mixture was then stirred for 1 hour, after which the solution was purged with $\mathrm{CO}_{2}$ and poured into acidic water to obtain the di(carboxylic acid). In this way, the product could be obtained with an improved yield of $63 \%$. 
Scheme 1. Synthesis of the monomers $1 L-B N-A C$ and $3 L-B N-A C$.

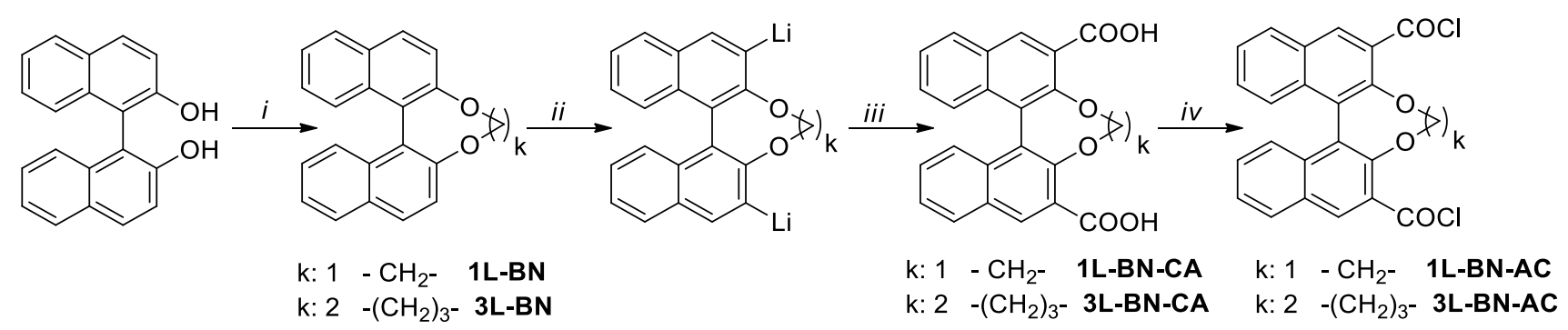

i. $\mathrm{CH}_{2} \mathrm{Br}_{2}$ or $\left(\mathrm{CH}_{2}\right)_{3} \mathrm{Br}_{2}$ respectively, $\mathrm{Nal}, \mathrm{K}_{2} \mathrm{CO}_{3}$, acetone, $60^{\circ} \mathrm{C}$; ii. $n-B u L i, E t_{2} \mathrm{O}, \mathrm{THF} ; \mathrm{iii}$. 1) $\left.\mathrm{CO}_{2} 2\right) \mathrm{H}^{+}, \mathrm{H}_{2} \mathrm{O}$; iv. Oxalyl chloride, toluene, $40^{\circ} \mathrm{C}$.

Lastly, the di(carboxylic acid) was converted to the di(acid chloride) (1L-BN-AC/3L-BN-AC) as described by Baruah et al.[51] This reaction was monitored by IR spectroscopy (See SI Figure S2). The reason why the starting material, (S)-1,1'-Binaphthalene-2,2'-diol, itself is not used for the top layer preparation is twofold: (1) the fenol derivative is not as reactive as the acid chloride derivative that is synthesized, for reaction with the amine monomers; (2) if the fenol derivative would be used to prepare the top layer, it would not be possible to study the influence of the dihedral angle on the membrane performance since the fenol functions are necessary for the synthesis of the alkyl bridge.

The amine 1,3,5TA.3 HCl was formed starting from the commercially available 3,5-dinitroaniline (Figure 5), using similar conditions as described by Takahira et al.[52] A THF/EtOAC (8:1 v:v) mixture was used as solvent to be able to precipitate the amine after the hydrogenation reaction, via the addition of concentrated $\mathrm{HCl}(37 \%)$ to yield the amine monomer in its stable salt-form.

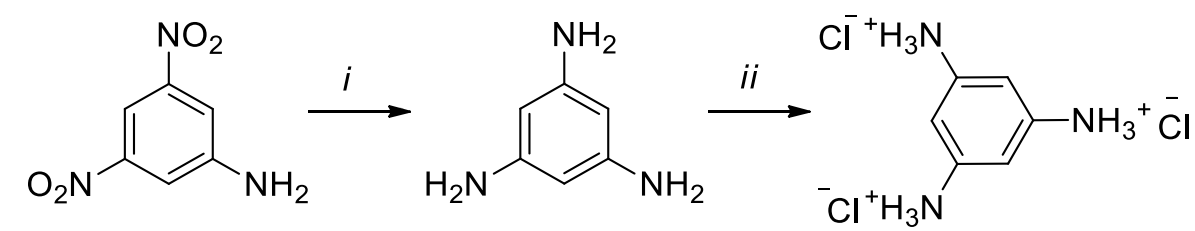

Figure 5. Schematic representation of the $1,3,5 \mathrm{TA} .3 \mathrm{HCl}$ synthesis. i. $\mathrm{H}_{2}, \mathrm{Pd} / \mathrm{C}, \mathrm{THF} / \mathrm{EtOAC}(8: 1), 40^{\circ} \mathrm{C} ; \mathrm{ii} . \mathrm{HCl}(37 \%), \mathrm{THF}, 0^{\circ} \mathrm{C}$

\subsection{Proof of principle}

Preliminary experiments were performed in small test vials in absence of a support. Therefore, aqueous solutions of varying amine concentrations were brought into contact with solutions of varying acid chloride concentrations in toluene. The optimal concentrations of the monomers and additives needed to be determined, to be able to prove that a network polymer could be formed at the interface of two immiscible solvents using this binaphthalene system. The formation of a polyamide layer was determined visually by the observation of a white layer at the interface. In the first film formation studies, the methylbridged binaphthalene di(acid chloride) (1L-BN-AC) was combined with the commercially available $1,2,4 \mathrm{TA} .2 \mathrm{HCl}$ (Figure 6). As a starting point, the first concentrations were based on the synthesis of the most commonly known top-layer formed from $m$-phenylene diamine (MPD) in water and trimesoyl chloride (TMC) dissolved in hexane (Table 1).[20] In doing so, it had to be considered that the common monomer system (e.g. TMC/MPD) combines a tri(acid chloride) with a diamine while the new system combines a di(acid chloride) with a triamine. To lower the surface tension, sodium dodecyl sulfate (SDS) is often added to the aqueous phase. TEA is added to the aqueous phase to facilitate the reaction and to react with the hydrogen chloride formed as side product. $[1,20]$ 


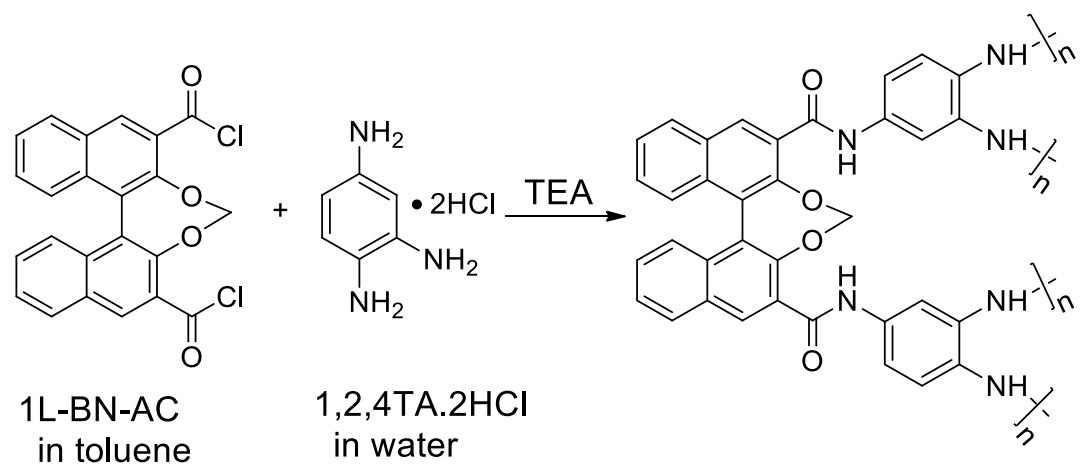

Figure 6. Schematic representation of the polymerization reaction.

See Table 1 for the concentrations used for the first film formation tests. It was noticed that upon addition of SDS to the aqueous phase, flocculation took place. Therefore, it was decided not to use SDS during the film formation tests. By adding SDS to the water phase the ionic strength of the solution increases which explains why flocculation takes place.

Table 1. Concentration of the commonly used system vs. the initial concentration used for the binaphthalene system.

Common System

\begin{tabular}{l|ll}
\hline Amine & $2 \mathrm{w} / \mathrm{v} \% \mathrm{MPD}$ & $1.33 \mathrm{w} / \mathrm{v} \% 1,2,4 \mathrm{TA} .2 \mathrm{HCl}$ \\
Acid chloride & $0.1 \mathrm{w} / \mathrm{v} \% \mathrm{TMC}$ & $0.15 \mathrm{w} / \mathrm{v} \%$ 1L-BN-AC \\
SDS & $0.5 \mathrm{w} / \mathrm{v} \%$ & $/$ \\
TEA & $2 \mathrm{w} / \mathrm{v} \%$ & $4 \mathrm{eq}$ \\
Organic phase & hexane & toluene
\end{tabular}

The addition of base to the aqueous phase has two important functions. First, the base serves as a catalyst for the reaction and as a pH-stabiliser, as in other IFP systems.[20] Second, the addition of base is also necessary to convert the amine salt to the free amine. Therefore, it was decided that when 1 eq of $1,2,4 \mathrm{TA} .2 \mathrm{HCl}$ was used, 2 eq of TEA were added to convert the amine salt. Additionally, 2 eq of TEA was added to catalyze the reaction and capture the released $\mathrm{HCl}$. For the conversion of the amine salt to the free amine, the addition of $\mathrm{NaOH}$ instead of TEA was also tested. However, it was decided to continue with TEA, as this addition did not give a substantial difference as well as making the process more cumbersome to perform. Due to solubility problems, the $\mathbf{1 L - B N - A C}$ concentration was limited to a maximum of $0.25 \mathrm{w} / \mathrm{v} \%$. However, as shown in Table 2, it was already possible to form a thin film using only $0.15 \mathrm{w} / \mathrm{v} \%$ of $1 \mathrm{~L}-\mathrm{BN}-\mathrm{AC}$. Initially, when using $1.33 \mathrm{w} / \mathrm{v} \%$ of $1,2,4 \mathrm{TA} .2 \mathrm{HCl}$ a nice film was formed (Table 1). However, when optimizing the amine concentration, the amount of amine used could be lowered to $0.2-0.5 \mathrm{w} / \mathrm{v} \%$ (Table 2). 


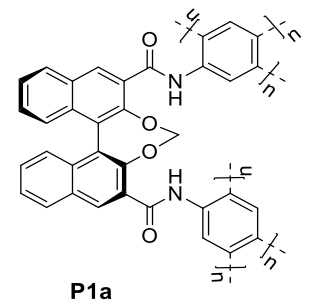

P1a

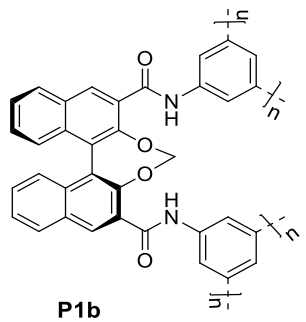

$\begin{array}{lr}\mathbf{k}=1 & \\ 1,2,4,5 T A & \mathbf{P 1 a} \\ 1,3,5 T A & \mathbf{P 1 b} \\ 1,2,4 T A & \mathbf{P 1 c} \\ 1,2,4 T A \& M P D & \mathbf{P 1 d}\end{array}$

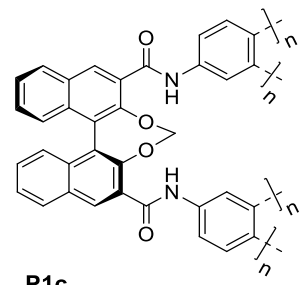

P1c

$$
\mathrm{k}=3
$$

1,2,4TA\&MPD P3d



P1d(k=1) / P3d (k=3)

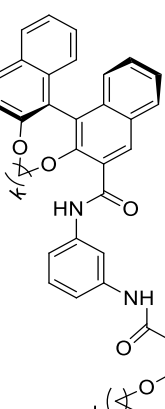

$k \Varangle_{0}^{\circ}$

$\mathrm{O}$

HN

Figure 7. The bridged binaphthalene polyamides Pxy formed by combining the binaphthalene di(acid chloride)s $1 L-B N-A C$ and $3 L-$ $B N-A C$ with the various amines monomers, with $x=1$ or 3 , when $1 L-B N-A C$ and $3 L-B N-A C$ are respectively used and $y=a, b, c$ or $d$ when $1,2,4,5 T A$ or $1,3,5 T A$ or 1,2,4TA or 1,2,4TA\&MPD are respectively used.

According to the vial test a nice film could still be obtained. In general, the films formed in the vial test with lower amounts of amine monomer looked even better than the ones formed with a higher concentration (Figure S16 - 17). The cracks formed at higher 1,2,4TA concentrations ( $0.5 \mathrm{w} / \mathrm{v} \%)$ can tentatively be explained by the fact that the film at the interface will be formed faster at higher concentrations, which on its turn limits the mass transport of the amine from the aqueous to the organic phase, yielding a thin and brittle layer that can easily break.

Table 2. Results of vial test in which the influence of the concentrations of amine and acid chloride were studied.*

\begin{tabular}{|c|c|c|c|}
\hline $\mathrm{C}_{1 \mathrm{~L}-\mathrm{BN}-\mathrm{AC}} \mathrm{C}_{1,2,4 \mathrm{~T}}$ & $0.2 \mathrm{w} / \mathrm{v} \%$ & $0.3 \mathrm{w} / \mathrm{v} \%$ & $0.5 \mathrm{w} / \mathrm{v} \%$ \\
\hline $0.15 \mathrm{w} / \mathrm{v} \%$ & + & + & \pm \\
\hline $0.25 \mathrm{w} / \mathrm{v} \%$ & + & + & + \\
\hline
\end{tabular}

In all vial tests, 4 eq of TEA was used, no SDS was added.

* ' + ' indicates a good, visible intact, polyamide film formation ' \pm ' indicates that cracks were visible in the polyamide film. These conclusions were based only on visual observations.

Due to the instability of the free amine, several precautions had to be taken during the film formation tests. The distilled water used for the aqueous phase was always purged for more than $2 \mathrm{~h}$ with argon while being sonicated to remove all oxygen present. Additionally, the amine solution was always the last solution to be prepared. Therefore, the amine was dissolved in the distilled water and sonicated, to ensure that it was completely dissolved, before adding the TEA. From the moment TEA was added, all following actions were performed as fast as possible. With the eye on scalability, when using argon, a blanket of inert gas will be present over the interfacial polymerization set-up ensuring that the amines will be stable long enough for the membrane preparation. Therefore, no problems with respect to the instability of the free amines are expected.

\subsection{Initial TFC membranes}

Based on the above observations, a series of 5 supported membranes were made on a XL-PI support. Before use, the XL-PI was rinsed excessively with methanol and stored in distilled water. Therefore, the 
probability of unreacted HDA being present in the support is assumed to be negligible. Although all measures have been taken to prevent the presence of unreacted HDA, there is still a small change that there is some unreacted HDA available. However, when mono- reacted HDA is present, this can also react with the acid chloride at the interface. In this way, the top layer is then covalently bound to the support layer which prevents the top layer from coming loose during filtration experiments in which swelling could be an issue. The $1 \mathrm{~L}-\mathrm{BN}-\mathrm{AC}$ concentration was kept constant at $0.15 \mathrm{w} / \mathrm{v} \%$ while the amine $(1,2,4 \mathrm{TA} .2 \mathrm{HCl})$ and TEA concentrations were varied according to Table 3. By combining $1 \mathrm{~L}-\mathrm{BN}-\mathrm{AC}$ with $1,2,4 \mathrm{TA} .2 \mathrm{HCl}$, the network polymer P1c was formed (see Figure 7).

Table 3. Summary of the concentrations used for the membrane synthesis.

\begin{tabular}{c|ccc} 
Membrane & Acid Chloride & Amine (w/v\%) & TEA (eq) \\
\hline M1 & & 0.2 & 3 \\
M2 & & 0.25 & 3 \\
M3 & $0.15 w / v \%$ & 0.3 & 2 \\
M4 & & 0.3 & 3 \\
M5 & & 0.3 & 4
\end{tabular}

To study how the variation in concentrations influences the top-layer formation, the membrane performance of these membranes was tested using Rose Bengal (RB) and Methyl Orange (MO) in distilled water (Figure 8, Supporting table 2 and 3).
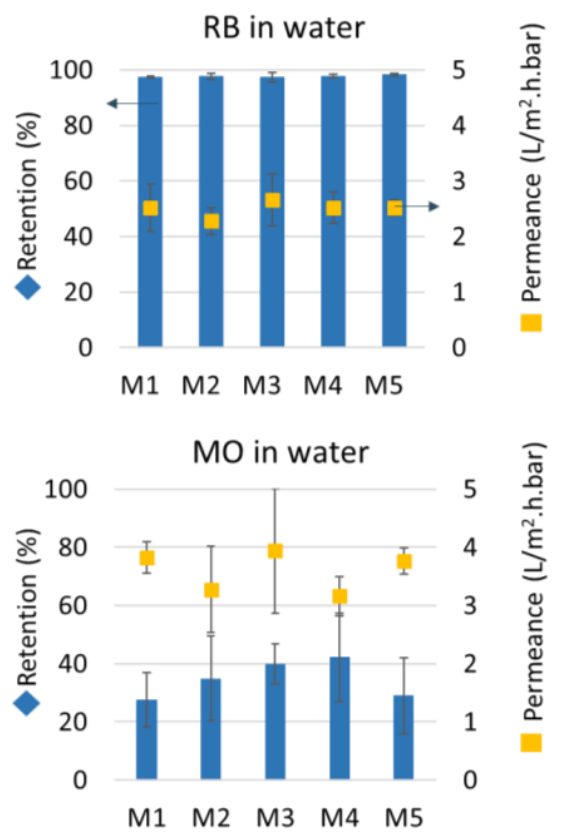

Figure 8. Overview of the filtration results. Top graph: Rose Bengal in water filtration, bottom graph: Methyl Orange in water filtration. Blue bars are the retention (left axis), the yellow dots are the permeance (right axis). 


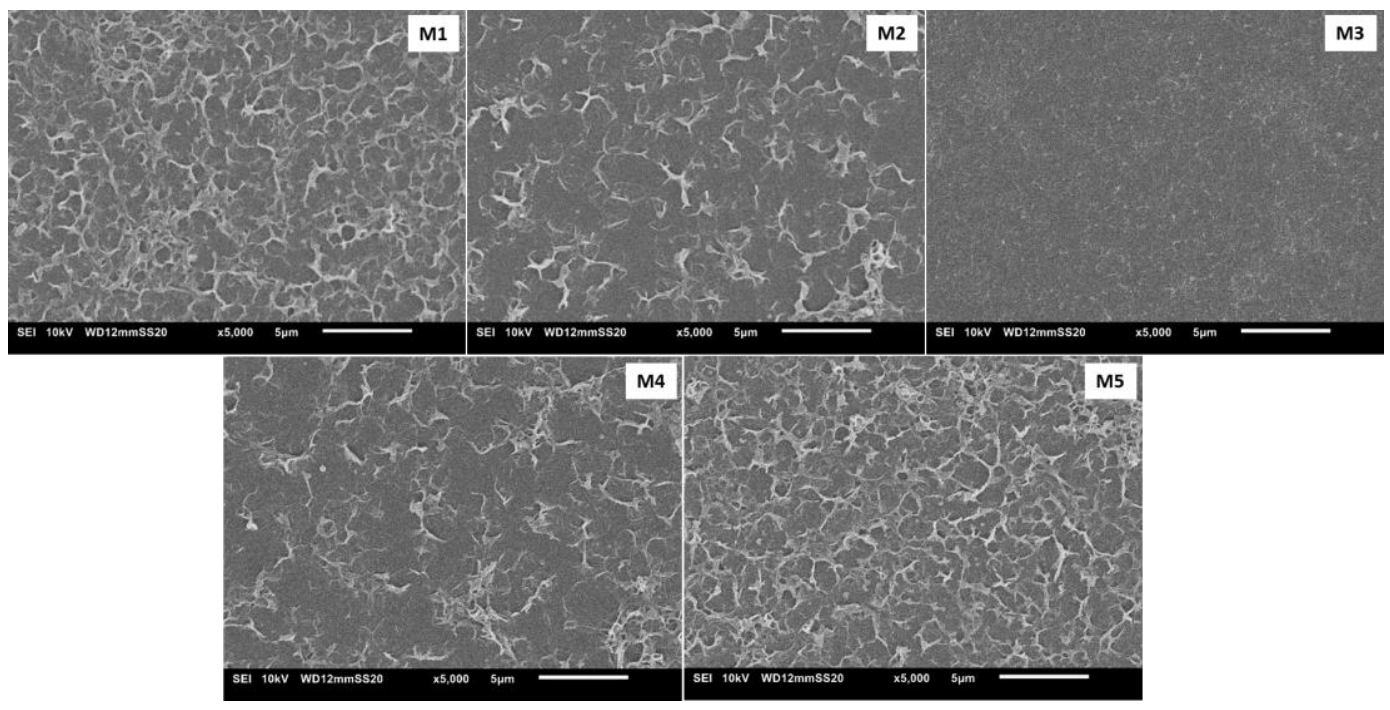

Figure 9. SEM top view images from membranes M1-M5 in which the preparation conditions were varied. The top-layer structure varies with varying concentration of amine and TEA.

All membranes showed retentions of more than $95 \%$ for RB but showed only retentions below $50 \%$ for $\mathrm{MO}$ (Figure 8). Since variating the concentrations of the monomers and base can have a large influence on the top-layer, SEM was used to study the morphology of the membranes. The morphology of the membranes significantly differed when varying the reaction conditions (Figure 9).

Since morphology differences of the TFC membranes could result in a different surface roughness, which on its turn could have effect on the membrane surface area and hence the permeance, TEM was used to characterize the cross-sections of the membranes M1-M5 (see supporting info, Figure S18). Although the surface roughness differs slightly, no direct relation can be made regarding the permeance since membrane $\mathrm{M} 3$ with the lowest surface roughness has the highest permeance. However, all membranes yielded good filtration results. From the filtration data of RB and $\mathrm{MO}$, it could be determined that the conditions used for the synthesis of M3 generated the best results. In addition, the conditions used for the preparation of membrane $\mathrm{M} 3$ require the smallest amount of base, hence with minimal interference on the film formation. Therefore, these conditions were thus used to study the influence of the various amine monomers and the influence of the length of the alkyl linker on the membrane performance.

\subsection{Variation of the amine monomer}

By combining 1L-BN-AC with 1,2,4TA.2HCl, a network polymer P1C is formed (Figure 7). The hypothesis is that by varying the amine monomer, and thus the number and position of amine functions, the structure of the polymer network changes. This is expected to have an influence on the density of the membrane and thus the membrane performance. In order to confirm this hypothesis, polymers P1a, P1b, and P1d were made via IFP and subsequently tested for their membrane performance. The new polymers were formed by combining the methyl-bridged $1 \mathrm{~L}-\mathrm{BN}-\mathrm{AC}$ with a $1: 1$ ratio of 1,2,4TA.2HCl and MPD (P1d), $1,3,5 \mathrm{TA} .3 \mathrm{HCl}(\mathbf{P} 1 \mathbf{b})$ and $1,2,4,5 \mathrm{TA} .4 \mathrm{HCl}(\mathbf{P} 1 \mathbf{a})$. In this way, the amount of amine functions is varied from 2.5 to 4 functions per monomer unit. The structures of the various polymers are shown in Figure 7 . The bridged binaphthalene polyamides Pxy are named according to the monomers used, with $x=1$ or 3 when $1 \mathrm{~L}-\mathrm{BN}-\mathrm{AC}$ and $3 \mathrm{~L}-\mathrm{BN}-\mathrm{AC}$ are respectively used and $\mathrm{y}=\mathrm{a}, \mathrm{b}, \mathrm{c}$ or $\mathrm{d}$ when $1,2,4,5$ TA or $1,3,5$ TA or $1,2,4$ TA or $1,2,4$ TA\&MPD are respectively used ( $a=$ highest amount of amine functionalities and $d=$ lowest amount of amine functionalities) 
Besides the above mentioned membranes, a reference membrane was prepared from MPD/TMC based PA. The membrane performance was determined by performing filtrations using RB in water, ethanol and ACN (Figure 10, Supporting table 2,4 and 5). It can be observed that when the amount of amine functional groups per monomer unit increases, the ACN permeance decreases.


Figure 10. Filtration results obtained when using membranes prepared with the various amines and with the standard TMC/MPD system as reference. Blue bars are the retention (left axis), the yellow dots are the permeance (right axis)

This is in line with the fact that a lower amount of amine functionalities results in a more open network structure. By increasing the number of amine functionalities, the number of branch points increases, the network tightens and the permeance thus decreases. This hypothesis is valid when the thickness of the active layers are all similar. Therefore, TEM images (see supporting info, Figure S19) were made from the cross-sections of the membranes which show that there is no significant difference in thickness. Moreover, the surface roughness for all membranes (P1a, P1b, $\mathbf{P 1 c}$ and $\mathbf{P} \mathbf{1 d}$ ) is similar, therefore, an equal contribution of the surface roughness to the membrane area and hence to the permeance is expected for all membranes. With exception of P1a, a similar trend can be observed for the ethanol filtrations. Despite the decrease in permeance, all obtained permeances for the binaphthalene-based membranes are an order of magnitude higher than the permeance obtained for the reference membrane. With exception of 
the membrane made of P1a, all binaphthalene-based membranes have a RB retention of more than $95 \%$ in all solvents. The best retention values are obtained for the filtration of RB in ACN. The MPD/TMC reference membrane has a retention that is always as good or better than the new membranes, however the permeance is always much lower. This could probably be explained by the tighter structure of the MPD/TFC reference membrane. When comparing the results of the ethanol and ACN filtrations to the water filtration, an opposite trend can be observed in terms of permeance. This trend cannot be explained by the tightness of the membranes since for water the highest amount of amine functions present gives the highest permeance. However, not only the number of functional amine groups varies but also the reactivity thereof. Based on the obtained results, it was hypothesized that the difference in permeance of water versus organic solvents, when using the various amines, could be attributed to a difference in reactivity of the amine functions present. A lower reactivity leads to the presence of a higher amount of unreacted amine and carboxylic acid functionalities in the final membrane film, which increases the membrane polarity. To validate this, the reactivity of the various amines was studied using gas chromatography - mass spectroscopy (GC-MS). In this experiment, the conversion of benzoyl chloride by reaction with the amine monomers was studied in the presence of 1,3,5-trimethoxybenzene as internal standard (Scheme 2).

Scheme 2. Reaction overview, used for the reactivity study performed via GC-MS

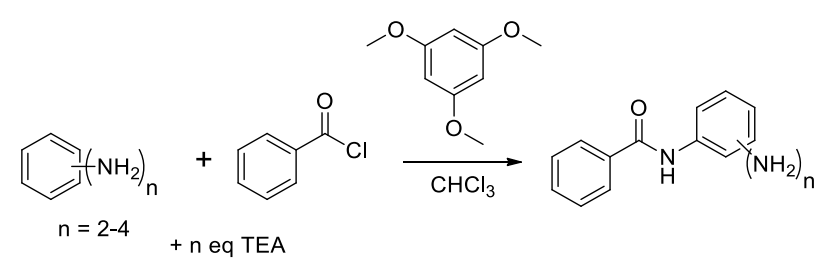

When plotting the obtained conversions for the various amine monomers, a clear difference in reactivity could be observed (Figure 11, Figure S20-S24 and supporting Table 1). When a 1:1 ratio of 1,2,4TA.2HCl with MPD was used as amine monomer, the highest conversion was obtained. This means that the polymer film that was formed, contains the lowest amount of free amine groups. Therefore, this film is less polar and has weaker interactions with the water molecules, which explains why the membrane has a lower water permeance while an increase in permeance can be observed for ACN. Besides, a less polar film, the increase in permeance for ACN could also be attributed to swelling. To have an indication of the extent in which swelling could occur, the Hildebrand (Hansen) solubility parameters can be calculated. The solubility parameters are widely used for correlating polymer solvent interactions and can be divided into three different contributions: polar $\left(\delta_{p}\right)$, dispersive $\left(\delta_{d}\right)$ and hydrogen bonding $\left(\delta_{h}\right)$. [53] For filtrations with acetonitrile based feeds, the solubility parameters were already calculated by Bastin et al.[11] The calculated data, relevant for this research, is summarized in table 4. The parameters for PA and ACN were calculated using the group contribution theory developed by van Krevelen et al. while for RB, the Fedors' group contribution is used, since this method is more suited for smaller molecules. Since, the solubility of a polymer in a solvent is largely determined by its chemical structure, solubility is favored when there is structural similarity. 
Table 4. Hildebrand solubility parameter*

\begin{tabular}{c|cccc} 
& $\delta_{d}$ & $\delta_{p}$ & $\delta_{h}$ & $\delta$ \\
\hline $\mathrm{PA}$ & 23.3 & 4.1 & 8.4 & 25.1 \\
$\mathrm{ACN}$ & 15.3 & 18.0 & 6.1 & 24.4 \\
$\mathrm{RB}$ & $/$ & $/$ & $/$ & 32.8
\end{tabular}

*The solubility parameter for RB is calculated via the Fedors' group contribution since this is more accurate for small molecules. So when, the solubility parameters of polymer and solvent are equal, the structures are similar and swelling is more likely to occur. Based on the values in table 4 , it could be said that swelling is likely to occur since there is only a small difference between $\delta_{P A}$ and $\delta_{A C N}$. However, two important stipulations need to be taken into account: (1) the calculations of the solubility parameters are based on the group contribution theory which means that no distinction can be made between the different amine monomers used and (2) the value for the $\delta$ is only valid when the reaction of the amines with the acid chloride is to full conversion. When Bastin et al. performed there calculations for PA, they assumed that the standard reaction of the MPD with TMC was to almost complete conversion and that the side reaction that could occur (hydroxylation of the acid chloride to carboxylic acid) doesn't affect the solubility parameter much. This means that the values for PA, in table 4 , are only a rough estimation since the amines that are used don't give a full conversion and the influence of the free amine functionalities isn't included.

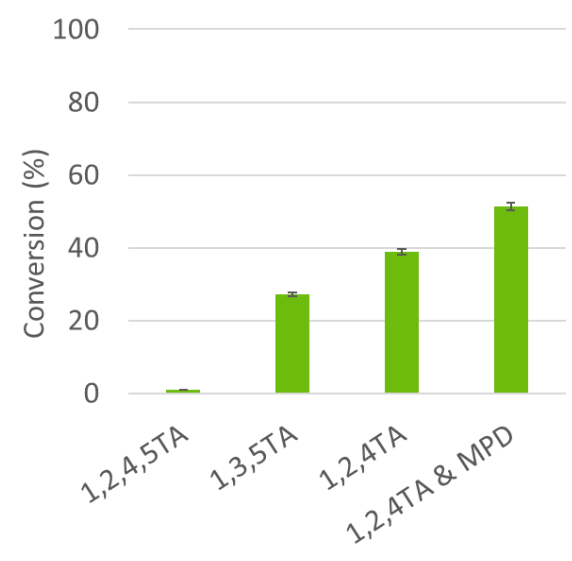

Figure 11. Conversion of benzoyl chloride for the various amine monomers.

Overall, following general trend can be observed: when going from the amine monomer with the lowest conversion to the amine with the highest conversion, the water permeance declines while the ACN permeance increases. This phenomenon can thus be attributed to the presence of more free amine and carboxylic functions in the end product. To corroborate this hypothesis the contact angle of water was measured (Figure 12). With exception of P1b, the contact angle increases going from P1a to P1d, which corroborates that the affinity for water is higher for P1a then for P1b, which can be explained by the higher amount of free amine groups that are present in the P1a membrane. 


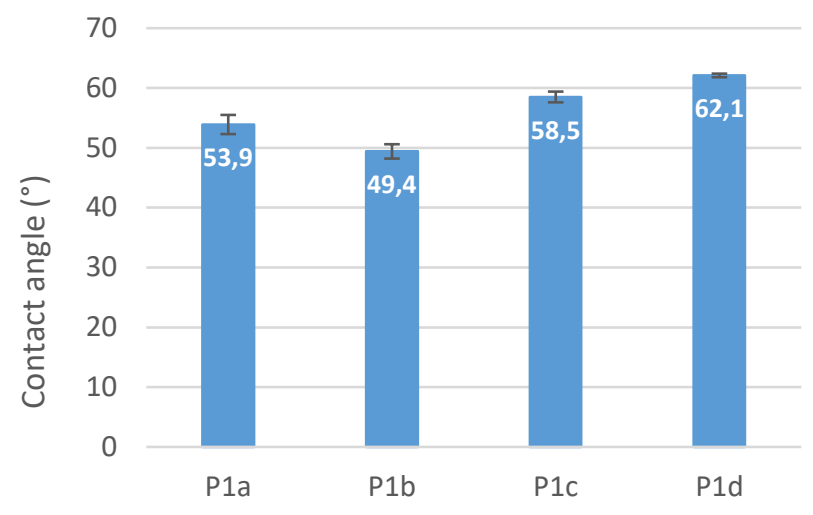

Figure 12.

In general, the permeance of the membranes made from P1d was 20x higher than the value obtained when using the reference membrane. The filtration data clearly show that the binaphthalene-based polymer films have a high retention in combination with a permeance that exceeds that from the standard TMC/MPD based polyamide film. When comparing the obtained RB in ACN filtration data for P1c and P1d with the currently commercially available membranes (Figure 12), it can be concluded that the newly obtained membranes exceed the membrane performance of all commercially available membranes.

\subsection{Influence of the binaphthalene linker}

Besides varying the choice of amine monomer, also the structure of the binaphthalene di(acid chloride) can be altered. By changing the length of the alkyl bridge, the dihedral angle in between the naphthalene unit changes between $49.1^{\circ}$ and $65.6^{\circ}$, when going from a methyl linker to a propyl linker, respectively. By applying this change in the monomer structure, it is expected that the macromolecular structure of the polymer film changes. This variation in macromolecular structure, on its turn, induces a change in the porosity and thus the permeance of the membrane. To study this, the two binaphthalene bridged monomers 1L-BN-AC and 3L-BN-AC were synthesized (Scheme 1) and combined with a 1:1 ratio of $1,2,4 \mathrm{TA} .2 \mathrm{HCl}$ and MPD as amine monomer, yielding polymers $\mathbf{P} \mathbf{1} \mathbf{d}$ and $\mathbf{P} \mathbf{3 d}$, respectively (Figure 7 ). This amine monomer system was chosen since it yielded the best membrane performance previously. 


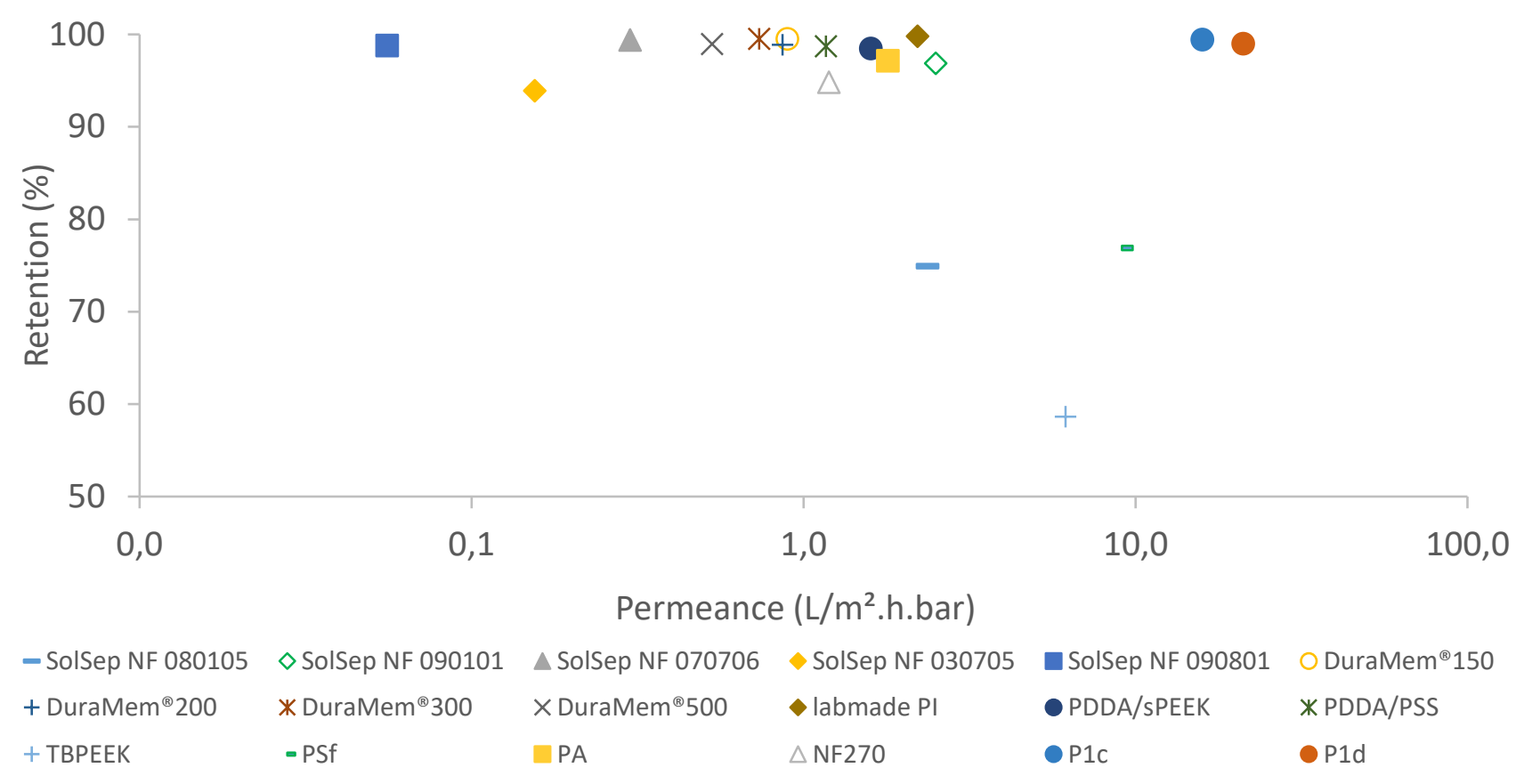

Figure 13. Robeson plot for RB in ACN, in which the ACN filtration data of all currently commercially available membranes is plotted together with the newly obtained data from membranes P1c and P1d

The membrane performance of both membranes was determined by performing filtrations using RB in water, ethanol and ACN (Figure 13, Supporting table 2,4 and 5). When comparing the membrane performance of the two polymers, it can clearly be observed that the methyl-bridged binaphthalenebased membrane $\mathbf{P} \mathbf{1} \mathbf{d}$ always showed the highest retention.


Figure 14. Filtration results of 1 L-BN-AC and $3 L-B N-A C$ when combined with a 1:1 ratio of 1,2,4TA.2HCl:MPD as amine monomer, yielding $\mathbf{P} 1 \mathbf{d}$ and $\mathbf{P} 3 \mathbf{d}$, respectively. Blue bars are the retention (left axis), the yellow dots are the permeance (right axis). 
Looking at the water and ethanol filtrations, the permeance is similar for both membranes P1d and P3d. For the ACN filtration, the obtained permeance is much higher when using $1 \mathrm{~L}-\mathrm{BN}-\mathbf{A C}$ as binaphthalene monomer than when using 3L-BN-AC. The propyl-bridged binaphthalene $\mathbf{3 L - B N}-\mathbf{A C}$ has the largest dihedral angle and yields to the lowest retention. Increasing the dihedral angle, by varying the length of the alkyl linker, gives thus probably a more open network structure. Based upon this, it can be concluded that by varying the length of the alkyl linker in between the naphthalene units, a change in filtration results is clearly observed. This indicates that the macromolecular structure of the polymer and thus the porosity of the membrane film can be varied by using a molecular adjustable monomer.

\section{Conclusions}

TFC SRNF membranes with a high permeance and RB retention could be formed using a bridged binaphthalene-based acid chloride monomer in combination with several rigid amine monomers. By changing the amount of amine functions per monomer unit and the length of the alkyl linker in the binaphthalene di(acid chloride), the macromolecular structure and thus the membrane performance could be tuned. The highest permeance for ACN could be obtained when using a $1: 1$ ratio of $1,2,4 \mathrm{TA} .2 \mathrm{HCl}$ and MPD, which has the lowest amine functions per monomer unit but the highest reactivity according to the conversion studies performed by GC-MS. In general, all binaphthalene-based membranes resulted in a higher permeance with a good retention in comparison to the standard TMC/MPD reference membrane. When comparing the methyl-bridged to the propyl-bridged binaphthalene monomer, the best filtration results were obtained for the methyl-bridged binaphthalene (1L-BN-AC). By combining a PIM-based concept with TFC membranes and a molecular adjustable monomer structure, the permeance of the ACN filtration could be increased by a factor of 20 , in comparison to the standard TFC membrane prepared from TMC/MPD.

\section{Acknowledgements}

We are grateful to the Fund for Scientific Research (FWO-Flanders - 1S22320N) and the Research Fund KU Leuven for financial support. M.T. is funded by FWO-SB. C.V.G. is funded by PDM (KU Leuven internal funds). TEM was funded by the Hercules - foundation (AKUL/13/19).

\section{References}

[1] S. Hermans, H. Mariën, C. Van Goethem, I.F. Vankelecom, Recent developments in thin film (nano)composite membranes for solvent resistant nanofiltration, Curr. Opin. Chem. Eng. 8 (2015) 45-54. https://doi.org/10.1016/j.coche.2015.01.009.

[2] P. Vandezande, L.E.M. Gevers, I.F.J. Vankelecom, Solvent resistant nanofiltration: separating on a molecular level, Chem. Soc. Rev. 37 (2008) 365-405. https://doi.org/10.1039/B610848M.

[3] P. Marchetti, M.F.J. Solomon, G. Szekely, A.G. Livingston, Molecular Separation with Organic Solvent Nanofiltration : A Critical Review, Chem. Rev. 114 (2014) 10735-10806. https://doi.org/10.1021/cr500006j.

[4] J. Geens, B. De Witte, B. Van Der Bruggen, Removal of API's (active pharmaceutical ingredients) from organic solvents by nanofiltration, Sep. Sci. Technol. 42 (2007) 2435-2449. https://doi.org/10.1080/01496390701477063.

[5] E.M. Rundquist, J. Pink, A.G. Livingston, C.J. Pink, A.G. Livingston, Organic solvent nanofiltration: A potential alternative to distillation for solvent recovery from crystallisation mother liquors, Green Chem. 14 (2012) 2197-2205. https://doi.org/10.1039/c2gc35216h.

[6] S. Li, C. Li, X. Song, B. Su, B. Mandal, B. Prasad, X. Gao, C. Gao, Graphene Quantum Dots-Doped Thin Film Nanocomposite Polyimide Membranes with Enhanced Solvent Resistance for Solvent- 
Resistant Nanofiltration, ACS Appl. Mater. Interfaces. 11 (2019) 6527-6540.

https://doi.org/10.1021/acsami.8b19834.

[7] B. Liang, H. Wang, X. Shi, B. Shen, X. He, Z.A. Ghazi, N.A. Khan, H. Sin, A.M. Khattak, L. Li, Z. Tang, Microporous membranes comprising conjugated polymers with rigid backbones enable ultrafast organic-solvent nanofiltration, Nat. Chem. 10 (2018) 961-967. https://doi.org/10.1038/s41557018-0093-9.

[8] S. Monjezi, M. Soltanieh, A.C. Sanford, J. Park, Polyaniline membranes for nanofiltration of solvent from dewaxed lube oil, Sep. Sci. Technol. 54 (2019) 795-802. https://doi.org/10.1080/01496395.2018.1512617.

[9] X.Q. Cheng, Y.L. Zhang, Z.X. Wang, Z.H. Guo, Y.P. Bai, L. Shao, Recent advances in polymeric solvent-resistant nanofiltration membranes, Adv. Polym. Technol. 33 (2014) 1-24. https://doi.org/10.1002/adv.21455.

[10] L. Liu, X. Wang, Y. Wang, L. Li, K. Pan, J. Yang, B. Cao, Preparation and characterization of asymmetric polyarylene sulfide sulfone (PASS) solvent-resistant nanofiltration membranes, Mater. Lett. 132 (2014) 11-14. https://doi.org/10.1016/j.matlet.2014.05.154.

[11] M. Bastin, K. Hendrix, I. Vankelecom, Solvent resistant nanofiltration for acetonitrile based feeds: A membrane screening, J. Memb. Sci. 536 (2017) 176-185. https://doi.org/10.1016/j.memsci.2017.05.003.

[12] P.G.N. Mertens, I.F.J. Vankelecom, P.A. Jacobs, D.E. De Vos, Gold nanoclusters as colloidal catalysts for oxidation of long chain aliphatic 1,2-diols in alcohol solvents, Gold Bull. 38 (2005) 157-162. https://doi.org/10.1007/BF03215255.

[13] M. Paul, S.D. Jons, Chemistry and fabrication of polymeric nanofiltration membranes: A review, Polymer. 103 (2016) 417-456. https://doi.org/10.1016/j.polymer.2016.07.085.

[14] S. Hermans, E. Dom, H. Mariën, G. Koeckelberghs, I.F.J. Vankelecom, Efficient synthesis of interfacially polymerized membranes for solvent resistant nanofiltration, J. Memb. Sci. 476 (2015) 356-363. https://doi.org/10.1016/j.memsci.2014.11.046.

[15] R. Oizerovich-Honig, V. Raim, S. Srebnik, Simulation of thin film membranes formed by interfacial polymerization, Langmuir. 26 (2009) 299-306. https://doi.org/10.1021/la9024684.

[16] C. Jiang, L. Tian, Y. Hou, Q.J.J. Niu, Nanofiltration membranes with enhanced microporosity and inner-pore interconnectivity for water treatment: Excellent balance between permeability and selectivity, J. Memb. Sci. 586 (2019) 192-201. https://doi.org/10.1016/j.memsci.2019.05.075.

[17] Z. Jiang, S. Karan, A.G. Livingston, Water Transport through Ultrathin Polyamide Nanofilms Used for Reverse Osmosis, Adv. Mater. 30 (2018) 1-7. https://doi.org/10.1002/adma.201705973.

[18] S.K. Das, P. Manchanda, K. Peinemann, Solvent-resistant triazine-piperazine linked porous covalent organic polymer thin-film nanofiltration membrane, Sep. Purif. Technol. (2019) 348358. https://doi.org/.1037//0033-2909.126.1.78.

[19] W. Xie, G.M. Geise, B.D. Freeman, H.-S. Lee, G. Byun, J.E. Mcgrath, Polyamide interfacial composite membranes prepared from $\mathrm{m}$-phenylene diamine, trimesoyl chloride and a new disulfonated diamine, J. Memb. Sci. 403-404 (2019) 152-161. https://doi.org/.1037//00332909.126.1.78.

[20] H. Mariën, L. Bellings, S. Hermans, I.F.J. Vankelecom, Sustainable Process for the Preparation of High-Performance Thin-Film Composite Membranes using lonic Liquids as the Reaction Medium, ChemSusChem. 9 (2016) 1101-1111. https://doi.org/10.1002/cssc.201600123.

[21] A.I. Schäfer, A.G. Fane, T.D. Waite, Nanofiltration: Principles and Applications, Elsevier Ltd, 2005.

[22] M. Mertens, C. Van Goethem, M. Thijs, G. Koeckelberghs, I.F.J. Vankelecom, Crosslinked PVDFmembranes for solvent resistant nanofiltration, J. Memb. Sci. 566 (2018) 223-230. https://doi.org/10.1016/j.memsci.2018.08.051.

[23] J.E. Cadotte, R.J. Petersen, R.E. Larson, E.E. Erickson, A new thin-film composite seawater reverse 
osmosis membrane, Desalination. 32 (1980) 25-31. https://doi.org/10.1016/S00119164(00)86003-8.

[24] J.E. Cadotte, K.E. Cobain, R.H. Forester, R.J. Petersen, Continued evaluation of in situ-formed condensation polymers for reverse osmosis membranes, 1975.

[25] M. Mulder, Basic principles of membrane technology, Kluwer Acad. Publ. (1996) 5. https://doi.org/10.1016/0376-7388(92)85058-Q.

[26] R.J. Petersen, Composite reverse osmosis and nanofiltration membranes, J. Memb. Sci. 83 (1993) 81-150. https://doi.org/10.1016/0376-7388(93)80014-O.

[27] G.Y. Chai, W.B. Krantz, Formation and characterization of polyamide membranes via interfacial polymerization, J. Memb. Sci. 93 (1994) 175-192. https://doi.org/10.1016/0376-7388(94)800065.

[28] N.B. McKeown, P.M. Budd, Exploitation of intrinsic microporosity in polymer-based materials, Macromolecules. 43 (2010) 5163-5176. https://doi.org/10.1021/ma1006396.

[29] N.B. Mckeown, Polymers of Intrinsic Microporosity, ISRN Mater. Sci. 2012 (2012) 1-16. https://doi.org/10.5402/2012/513986.

[30] P.M. Budd, B.S. Ghanem, S. Makhseed, N.B. Mckeown, K.J. Msayib, E. Tattershall, Polymers of intrinsic microporosity (PIMs): robust, solution-processable, organic nanoporous materials, Chem. Commun. (2004) 230-231.

[31] N.B. Mckeown, The synthesis of polymers of intrinsic microporosity ( PIMs ), 60 (2017). https://doi.org/10.1007/s11426-017-9058-x.

[32] M.L. Jue, C.S. McKay, B.A. Mccool, M.G. Finn, R.P. Lively, U. States, Effect of Nonsolvent Treatments on the Microstructure of PIM-1, Macromolecules. 48 (2015) 5780-5790. https://doi.org/10.1021/acs.macromol.5b01507.

[33] D. Fritsch, P. Merten, K. Heinrich, M. Lazar, M. Priske, High performance organic solvent nanofiltration membranes : Development and thorough testing of thin film composite membranes made of polymers of intrinsic microporosity ( PIMs ), J. Memb. Sci. 401-402 (2012) 222-231. https://doi.org/10.1016/j.memsci.2012.02.008.

[34] M.F. Jimenez-Solomon, Q. Song, K.E. Jelfs, M. Munoz-Ibanez, A.G. Livingston, Polymer nanofilms with enhanced microporosity by interfacial polymerization, Nat. Mater. (2016) 1-10. https://doi.org/10.1038/nmat4638.

[35] P.M. Budd, N.B. Mckeown, D. Fritsch, Polymers of Intrinsic Microporosity ( PIMs ): High Free Volume Polymers for Membrane Applications, 1 (2006) 403-405. https://doi.org/10.1002/masy.200651356.

[36] P.H.H. Duong, D.H. Anjum, K.V. Peinemann, S.P. Nunes, Thin porphyrin composite membranes with enhanced organic solvent transport, J. Memb. Sci. 563 (2018) 684-693. https://doi.org/10.1016/j.memsci.2018.04.038.

[37] Y.J. Tang, Z.L. Xu, S.M. Xue, Y.M. Wei, H. Yang, Tailoring the polyester/polyamide backbone stiffness for the fabrication of high performance nanofiltration membrane, J. Memb. Sci. 541 (2017) 483-491. https://doi.org/10.1016/j.memsci.2017.07.033.

[38] Y.J. Tang, H. Ding, Z.L. Xu, B.Q. Huang, High-performance composite nanofiltration membranes fabricated via ternary mixture: Complementary preponderance of the fluorine-containing monomer 2,2'-bis(1-hydroxyl-1-trifluoromethyl-2,2,2-triflutoethyl)-4,4'-methylene dianiline and the rigid monomer bi, J. Appl. Polym. Sci. 135 (2018) 1-9. https://doi.org/10.1002/app.46482.

[39] H. Mariën, I.F.J. Vankelecom, Transformation of cross-linked polyimide UF membranes into highly permeable SRNF membranes via solvent annealing, J. Memb. Sci. 541 (2017) 205-213. https://doi.org/10.1016/j.memsci.2017.06.080.

[40] K. Vanherck, A. Cano-odena, G. Koeckelberghs, T. Dedroog, I. Vankelecom, A simplified diamine crosslinking method for PI nanofiltration membranes, J. Memb. Sci. 353 (2010) 135-143. 
https://doi.org/10.1016/j.memsci.2010.02.046.

[41] P. Vandezande, L.E.M. Gevers, J.S. Paul, I.F.J. Vankelecom, P.A. Jacobs, High throughput screening for rapid development of membranes and membrane processes, J. Memb. Sci. 250 (2005) 305310. https://doi.org/10.1016/j.memsci.2004.11.002.

[42] G. Koeckelberghs, S. Sioncke, T. Verbiest, A. Persoons, C. Samyn, Synthesis and properties of chiral helical chromophore-functionalised polybinaphthalenes for second-order nonlinear optical applications, Polymer. 44 (2003) 3785-3794. https://doi.org/10.1016/S0032-3861(03)00356-2.

[43] G. Koeckelberghs, M. Vangheluwe, I. Picard, L. De Groof, T. Verbiest, A. Persoons, C. Samyn, Synthesis and properties of new chiral donor-embedded polybinaphthalenes for nonlinear optical applications, Macromolecules. 37 (2004) 8530-8537. https://doi.org/10.1021/ma0491816.

[44] M. Agnes, A. Nitti, D.A. Vander Griend, D. Dondi, D. Merli, D. Pasini, A chiroptical molecular sensor for ferrocene, Chem. Commun. 52 (2016) 11492-11495. https://doi.org/10.1039/c6cc05937f.

[45] L. Pu, 1,1'-Binaphthyl dimers, oligomers, and polymers: molecular recognition, asymmetric catalysis, and new materials, Chem. Rev. 98 (1998) 2405-2494. https://doi.org/10.1021/cr970463w.

[46] D. Pasini, A. Nitti, Recent Advances in Sensing Using Atropoisomeric Molecular Receptors., Chirality. 28 (2016) 116-123. https://doi.org/10.1002/chir.

[47] W. Dujardin, C. Van Goethem, Z. Zhang, R. Verbeke, M. Dickmann, W. Egger, E. Nies, I. Vankelecom, G. Koeckelberghs, Fine-tuning the molecular structure of binaphthalene polyimides for gas separations, Eur. Polym. J. 114 (2019) 134-143. https://doi.org/10.1016/j.eurpolymj.2019.02.014.

[48] M.M. Mulunda, C. Van Goethem, Z. Zhang, I. Vankelecom, G. Koeckelberghs, Influence of the molecular structure of polybinaphthalene on the membrane separation performance, Eur. Polym. J. (2018) 248-254.

[49] H. Deussen, E. Hendrickx, C. Boutton, D. Krog, K. Clays, K. Bechgaard, Novel Chiral Bis-dipolar 6,6' -Disubstituted Binaphthol Derivatives for Second-Order Nonlinear Optics : Synthesis and Linear and Nonlinear Optical Properties, J. Am. Chem. Soc. 1337 (1996) 6841-6852.

[50] T.R. Wu, L.X. Shen, J.M. Chong, Asymmetric allylboration of aldehydes and ketones using 3,3'disubstitutedbinaphthol-modified boronates, Org. Lett. 6 (2004) 2701-2704.

[51] P.K. Baruah, R. Gonnade, P.R. Rajamohanan, H.J. Hofmann, G.J. Sanjayan, BINOL-based foldamers - Access to oligomers with diverse structural architectures, J. Org. Chem. 72 (2007) 5077-5084. https://doi.org/10.1021/jo070396y.

[52] I. Takahira, H. Fuchida, S. Tabata, N. Shindo, S. Uchinomiya, I. Hamachi, A. Ojida, Design of a binuclear $\mathrm{Ni}(\mathrm{II})$-iminodiacetic acid (IDA) complex for selective recognition and covalent labeling of His-tag fused proteins, Bioorganic Med. Chem. Lett. 24 (2014) 2855-2858. https://doi.org/10.1016/j.bmcl.2014.04.096.

[53] D.W. van Krevelen, K. te Nijenhuis, Properties of Polymers, Fourth, co, Elsevier, Amsterdam, 2009. 